\title{
Predictive power dispatch through negotiated locational pricing
}

\author{
Joseph Warrington, Member, IEEE, Sébastien Mariéthoz, Member, IEEE, \\ Colin N. Jones, Member, IEEE, Manfred Morari, Fellow, IEEE.
}

\begin{abstract}
A predictive mechanism is proposed in order to reduce price volatility linked to large fluctuations from demand and renewable energy generation in competitive electricity markets. The market participants are modelled as price-elastic units, price-inelastic units, and storage operators. The distributed control algorithm determines prices over a time horizon through a negotiation procedure in order to maximize social welfare while satisfying network constraints. A simple flow allocation method is used to assign responsibility for constraint violations on the network to individual units and a control rule is then used to adjust nodal prices accordingly. Such a framework is appropriate for the inclusion of aggregated household appliances or other 'virtual' market participants realized through smart grid infrastructure. Results are examined in detail for a 4-bus network and then success is demonstrated for a densely-populated 39-bus network. Formal convergence requirements are given under a restricted subset of the demonstrated conditions. The scheme is shown to allow storage to reduce price volatility in the presence of fluctuating demand.
\end{abstract}

Index Terms-Price-based control, model predictive control, dual decomposition, wind power, power flow allocation, electricity pricing, nodal pricing, locational marginal pricing

\section{INTRODUCTION}

Since the liberalization of electricity markets, the simple top down approach to power dispatch has been sacrificed for one in which operators compete to provide the most cost-efficient power. Under such a system an auction takes place in advance for every time slot of the following day in order to determine a scheduled price per MWh. However since this auction has become heavily distorted in some countries by the preferential treatment of an increasing contribution of wind power, the current system increasingly fails to provide a sensible clearing price. Other factors leading to price spikes include higherthan-expected demand and unscheduled supply outages [1]. Importantly, the increasing use of wind power has also meant a larger use of operating reserves to deal with the power variation [2]. This means larger operating margins have to be set aside in conventional generators, which adds expense and reduces efficiency in terms of emissions per MWh.

The optimal dispatch problem has been studied since the mid-20th century by grid operators, and much standard literature on the subject exists [3], [4]. Control of the network by price signals has been studied since the work of Schweppe et al. in 1980 [5]. Glavitsch and Alvarado [6], [7] have studied

This work was supported by the European Commission Research Project FP7-ICT-249096 Price-based Control of Electrical Systems (E-PRICE).

J.C. Warrigton, S. Mariéthoz, C.N. Jones and M. Morari are with the Automatic Control Laboratory, ETH Zürich, Physikstrasse 3, CH-8092 Zürich, Switzerland (e-mail: warrington@control.ee.ethz.ch) grid control using price mechanisms and points out that while it may be possible to infer convex cost functions of generators on the network, and therefore solve a central quadratic program to derive optimal nodal prices, time varying costs and shut-down/start-up events represent a major problem. This is because all plants have costs associated with maintenance and large changes in output which cannot be factored into a central price controller; indeed it is those smaller generators used to match peaks while the price is fluctuating most who are most concerned with when to start up or shut down.

There has been recent interest in decentralizing the optimal dispatch problem; [8] gives an account of the recent literature. The dispatch problem is an optimization with a separable cost function but with coupling constraints beween the nodes due to the need to satisfy each load and respect line flow limits. The solution can be approached using primal or dual decomposition. The problem with the primal decomposition approach is that it leads to setting power outputs from each generator to satisfy loads. Furthermore if we were to attempt a subgradient technique we would have to rely on Lagrange multipliers to be returned from the local optimizations, which in a market populated by selfish agents is not realistic. Neither of these aspects are desirable in a competitive, price-based environment.

More appropriately, dual decomposition (Lagrangian relaxation), where prices (Lagrange multipliers) are updated based on constraint violation until feasibility is reached, as demonstrated by [9]. Wang et al. [10] showed how a scheduled unit commitment problem could be solved by iterating price and schedule updates, however the number of Lagrange multipliers in the decomposition meant that the individual market participants were made to perform obscure or nonintuitive optimizations; this is a practical detraction from such a scheme.

For real-time control (on the minutes-seconds scale), a simpler strategy is required. Jokić et al. [11] have studied dynamic optimal dispatch and designed a continuous-time decentralized linear complementarity controller to satisfy power demands and line constraints, and proved convergence to the optimal solution under strong assumptions. They took minimization of total costs to price-sensitive units as the objective, on the grounds that this can be interpreted as a maximization of social welfare [12]. The limitation however was that in practice relying on real-time frequency droop as a control signal assumes sufficient total machine inertia that grid synchronism is not lost during the control action; no guarantees are made in this respect. The scheme also employs a model in which 
frequency drops are local; it would be necessary for a real control formulation to take the global system frequency as an input to the controller.

The scheme in this paper is presented as an alternative strategy to real-time price-based frequency control, with the assumption that reserve services correcting frequency on very short timescales would remain. It aims to match the pricesensitive power with the predicted amount of price-insensitive power at each step over a receding horizon, while respecting all network constraints. Time steps are on the order of minutes. At every time step, the negotiation scheme results in a new generation schedule exploiting the latest predictions, with the assumption that inelastic loads and supplies can be predicted well. The optimization action at a single time step of such a scheme is described in this paper, with implications for receding horizon control to be explored in future work.

\section{SYSTEM MODEL}

\section{A. Network}

The lines in the electrical network are assumed to have small resistance in comparison to their inductance. Consequently, a network of lines connecting $n$ nodes is defined here solely by a per-unit susceptance matrix $\Sigma \in \mathbb{S}_{+}^{n}$. Each element $\sigma_{i j}$ is the susceptance of the line connecting node $i$ to node $j$. $\sigma_{i j}=0$ implies nodes $i$ and $j$ are not connected directly, although all nodes are at least connected via lines to other nodes. There are no unpopulated leaf nodes on the network graph. Relative phase angles between buses are assumed to be small. Under these assumptions there are no line losses, voltages are constant across the network, and the per-unit power flow in steady state in the direction $i$ to $j$ is given by:

$$
P_{i j}=\sigma_{i j}\left(\delta_{j}-\delta_{i}\right)
$$

where $\delta_{i}$ is the phase angle at node $i$. Under this assumption, given a set of power injections due to generators and a matching set of loads, the real power flow can be determined uniquely from $\Sigma$, see [13] for details. Reactive power transfers are outside the scope of this paper.

\section{B. Networked units}

Many diverse consumers and producers are connected to real electricity networks, as shown in Fig. 1. In this study, we assume that they can be modelled using the three unit types described mathematically below, either singly or in combination. In this scheme, units do not consider their effects on the network, but provide selfish optimizations for their planned power draw or outputs given prices resulting from a negotiation.

Price-elastic: These units can be generators or consumers, and participant $i$ performs an optimization of the following form over a time horizon of $T$ steps:

$$
\begin{aligned}
& \min _{\mathbf{p}_{i}} J_{i}^{\mathrm{e}}\left(\mathbf{p}_{i}\right)-\boldsymbol{\lambda}_{i}^{\mathrm{T}} \mathbf{p}_{i}:=\sum_{k=1}^{T}\left[\tilde{J}_{i}\left(p_{i k}\right)+\alpha_{i}\left(\Delta p_{i k}\right)^{2}-\lambda_{i k} p_{i k}\right] \\
& \text { s. t. } \underline{p}_{i} \leq p_{i k} \leq \bar{p}_{i}, \quad \forall k=1, \ldots, T, \\
& \underline{\Delta p}_{i} \leq \Delta p_{i k} \leq \overline{\Delta p}_{i}, \quad \forall k=1, \ldots, T .
\end{aligned}
$$

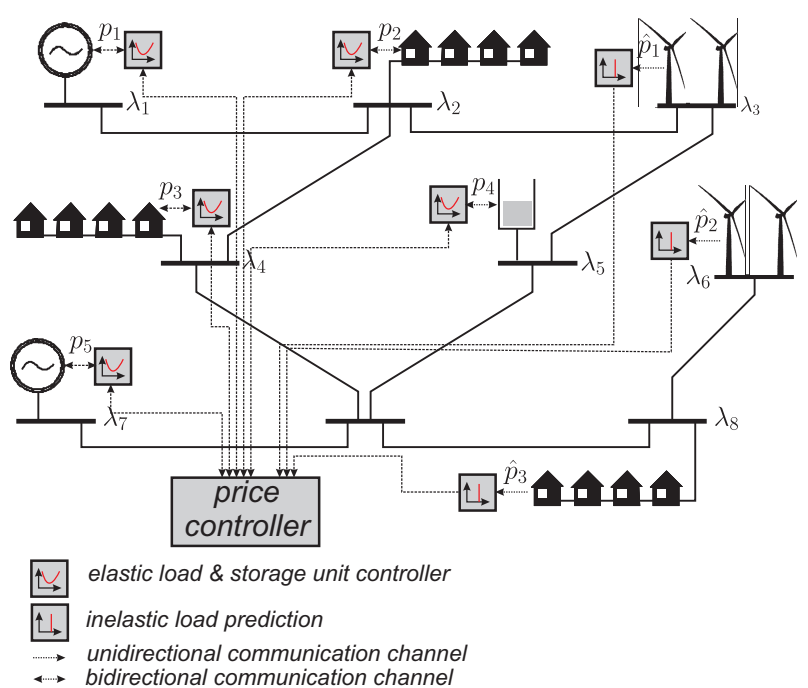

Fig. 1. Network supporting a diverse population of consumers and producers. The conventional generators will be price-elastic, wind farms may be inelastic, and households may have both inelastic and elastic demand components.

$\mathbf{p}_{i}:=\left[p_{i 1} \ldots p_{i T}\right]^{\mathrm{T}}$ is the vector of power outputs at each time step, and $\boldsymbol{\lambda}_{i}:=\left[\lambda_{i 1} \ldots \lambda_{i T}\right]^{\mathrm{T}}$ is the price schedule over the horizon, which is given for each optimization, with $\lambda_{i k}$ the price per unit of electrical energy, scaled for the length of the time step used. $\tilde{J}_{i}(\cdot)$ is the static cost function of the participant; here we assume that the cost can be represented adequately by a quadratic function, i.e. $\tilde{J}_{i}(p):=$ $a_{i}+b_{i} p+\frac{1}{2} c_{i} p^{2}$. For generators this comprises fuel and other variable costs, and for consumers this represents the utility of the power consumed. Real power $p_{i k}$ is defined as positive when injected into the grid. $\alpha_{i}$ is a penalty for changes power output, which are represented by $\Delta p_{i k}:=p_{i k}-p_{i[k-1]}$. The power at the time the optimization is performed is $p_{i 0} \cdot \underline{p}_{i}$ and $\Delta p_{i}$ are the (negative) lower bounds on power output and change in power output respectively, and $\bar{p}_{i}$ and $\overline{\Delta p}_{i}$ are upper bounds. Scheduling decisions, such as shut-down/startup or load shifting, are outside the scope of this study. Here $m_{\mathrm{e}}$ elastic units are present.

Price-inelastic: These units have a predicted power profile $\hat{\mathbf{p}}_{l}:=\left[\hat{p}_{l 1} \ldots \hat{p}_{l T}\right]^{\mathrm{T}}$ that cannot be influenced by price. Examples include inelastic demand and any wind power not taking part in active pricing mechanisms. Flows out of the grid are defined as positive. Since their power profile cannot be changed it is not meaningful to define a cost function for them. Here $m_{\mathrm{i}}$ inelastic units are present. Until convergence to $\hat{\mathbf{p}}_{l}$, values of these flows are denoted $\tilde{\mathbf{p}}_{l}:=\left[\tilde{p}_{l 1} \ldots \tilde{p}_{l T}\right]^{\mathrm{T}}$.

Storage: In general the aim of operating storage is to profit from selling power at a higher price than that for which it has been bought. The optimization of the storage level schedule for such a unit $i$ is formulated as follows:

$$
\begin{array}{ll}
\min _{\mathbf{s}_{i}} & J_{i}^{\mathrm{s}}\left(\mathbf{s}_{i}\right)+\boldsymbol{\lambda}_{i}^{\mathrm{T}} \Delta \mathbf{s}_{i}:=\sum_{k=1}^{T} \gamma_{i}\left(s_{i k}-s_{i}^{\mathrm{mid}}\right)^{2}+\lambda_{i k} \Delta s_{i k} \\
\text { s. t. } & \underline{p}_{i} \leq s_{i k}-s_{i[k-1]} \leq \bar{p}_{i} \quad \forall k=1, \ldots, T \\
& \underline{s}_{i} \leq s_{i k} \leq \bar{s}_{i} \quad \forall k=1, \ldots, T-1 \\
& s_{i T}=s_{i 0} .
\end{array}
$$


where $\mathbf{s}_{i}:=\left[s_{i 1} \ldots s_{i T}\right]^{\mathrm{T}}$ is the vector of storage levels for the unit over the horizon, $\Delta s_{i k}:=s_{i k}-s_{i[k-1]}, \gamma_{i}$ is a penalty for deviating from the midpoint $s_{i}^{\text {mid }}$ of the storage's capacity, $\underline{p}_{i}$ and $\bar{p}_{i}$ are as above, and $\underline{s}_{i}$ and $\bar{s}_{i}$ are the storage limits. The storage level at the time the optimization is performed is $s_{i 0}$, and the last constraint ensures that storage is not simply emptied at the end of the horizon: although this would maximize income over this time horizon, it would only do so at the expense of future income after time step $T$. Depending on the characteristics of the storage unit, modifications to the form given may be appropriate; the form given here is assumed for simplicity. In any case the optimization should return a power output profile from the optimal storage vector $\mathbf{s}_{i}$ found, given (according to the price-elastic sign convention) by:

$$
p_{i k}=-\Delta s_{i k}=s_{i[k-1]}-s_{i k}
$$

Here $m_{\mathrm{S}}$ storage units are present. Note that storage and priceelastic units perform differing optimizations but in each case return a vector of power outputs given a vector of prices.

\section{Global optimization}

The global objective of the scheme to be solved using the algorithm is, over a time horizon of $T$ intervals, to minimize total cost while satisfying power balance and network flow constraints:

$$
\begin{aligned}
& \min _{\mathbf{p}_{1}, \ldots, \mathbf{p}_{m_{\mathrm{e}}+m_{\mathrm{s}}}} \sum_{i=1}^{m_{\mathrm{e}}} J_{i}^{\mathrm{e}}\left(\mathbf{p}_{i}\right)+\sum_{i=1}^{m_{\mathrm{s}}} J_{i}^{\mathrm{s}}\left(\mathbf{p}_{i}\right) \\
& \text { s. t. } \underline{P}_{e} \leq P_{e k} \leq \bar{P}_{e}, \quad \forall e \in \mathcal{E}, \forall k=1, \ldots, T \text {, } \\
& \sum_{i=1}^{m_{\mathrm{e}}+m_{\mathrm{s}}} p_{i k}=\sum_{l=1}^{m_{\mathrm{i}}} \hat{p}_{l k}, \quad \forall k=1, \ldots, T, \\
& \tilde{p}_{l k}=\hat{p}_{l k}, \quad l=1, \ldots, m_{\mathrm{i}}, \forall k=1, \ldots, T
\end{aligned}
$$

where $\mathcal{E}:=\left\{(i, j): \sigma_{i j} \neq 0\right\}$, so that $P_{e k}$ is the appropriate flow $P_{i j}$ as defined in (1) at time step $k . \underline{P}_{e}$ and $\bar{P}_{e}$ are the lower and upper limits for real power flow in line $e \in \mathcal{E}$; for symmetrical bi-directional lines $\underline{P}_{e}=-\bar{P}_{e}$. Condition $(5 \mathrm{~d})$ matches the power drawn by each price-inelastic unit to its predicted value, as explained in section III. Note the abuse of notation for cleanness, which groups the $m_{\mathrm{e}}$ elastic generators in summations alongside the $m_{\mathrm{s}}$ storage units.

The aim is to set prices $\boldsymbol{\lambda}_{i}$ such that the power outputs $\mathbf{p}_{i}$ minimize the cost function under the profit-maximization strategies of the participants. If the Lagrangian of this problem were written out and separated, the task would be to find a set of prices that approximate the combination of optimal Lagrange multipliers arising from the constraints, and therefore give optimal 'selfish' behaviour for each participant.

\section{Control Scheme}

\section{A. Summary of the negotiation procedure}

The negotiation contains six processes, which are illustrated in Fig. 2.

1) Price initialization (price controller): An initial set of nodal prices $\boldsymbol{\lambda}_{0}:=\boldsymbol{\lambda}_{i}(0)$ for the time horizon is passed to each participant on node $i$.
2) Local optimization (price-sensitive units): Given their current price vector at round $j$, denoted $\boldsymbol{\lambda}_{i}(j)$, priceelastic units and storage operators perform local optimizations and commit to an optimal power schedule $\mathbf{p}_{i}$. This is a draft schedule that is refined as prices are adjusted in later negotiation rounds, but at each iteration $\mathbf{p}_{i}$ should be a feasible output commitment, such that if there are no further price changes the output will be exactly $\mathbf{p}_{i}$.

3) Power flow computation: Flows are determined, either centrally using the procedure in [13], or in a distributed manner, see the Appendix. Mismatches are accommodated until convergence by sharing the mismatch between the inelastic generators or loads on the network. This can be done proportionally (equal percentage mismatch):

$$
\tilde{p}_{l k}(j)=\hat{p}_{l k}+\frac{\left|\hat{p}_{l k}\right|}{\sum_{i=1}^{m_{\mathrm{i}}}\left|\hat{p}_{i k}\right|}\left(\sum_{i=1}^{m_{\mathrm{e}}+m_{\mathrm{s}}} p_{i k}(j)-\sum_{i=1}^{m_{\mathrm{i}}} \hat{p}_{i k}(j)\right)
$$

or equally (equal absolute mismatch):

$$
\tilde{p}_{l k}(j)=\hat{p}_{l k}+\frac{1}{m_{\mathrm{i}}}\left(\sum_{i=1}^{m_{\mathrm{e}}+m_{\mathrm{s}}} p_{i k}(j)-\sum_{i=1}^{m_{\mathrm{i}}} \hat{p}_{i k}(j)\right)
$$

with little difference in performance.

4) Responsibility assignment (price controller): Imbalance responsibilities $\phi_{i, l k}(j) \geq 0$ and line limit violations responsibilities $\Phi_{i, e k}(j) \geq 0$ are allocated to the priceelastic and storage units on the network, using an algorithm due to Bialek [14]. Such an algorithm requires only an assumption of thorough power mixing at each node, and therefore does not discriminate against any participant. Details are given in section III-B. Assignment gives a set of $\Phi_{i, e k}(j)$ and $\phi_{i, l k}(j)$ such that at negotiation stage $j$ :

$$
\begin{aligned}
& \sum_{i=1}^{m_{\mathrm{e}}+m_{\mathrm{s}}} \phi_{i, l k}(j)=1, \forall(l, k) \text { where } p_{l k} \neq \hat{p}_{l k}, \\
& \sum_{i=1}^{m_{\mathrm{e}}+m_{\mathrm{s}}} \Phi_{i, e k}(j)=1, \\
& \quad \forall(e, k) \text { where }\left(P_{e k}>\bar{P}_{e}\right) \vee\left(P_{e k}<\underline{P}_{e}\right) .
\end{aligned}
$$

5) Price updates (price controller): According to the results of steps 3 and 4, price feedback is sent to each participant according to the line overflows and load mismatches it has caused, for each time period $k$ :

$$
\begin{aligned}
\lambda_{i k}(j+1):= & \lambda_{i k}(j) \\
& +\sum_{l=1}^{m_{\mathrm{i}}} \kappa_{\mathrm{L}}\left(\hat{p}_{l k}, \tilde{p}_{l k}(j), \phi_{i, l k}(j)\right) \\
& +\sum_{e \in \mathcal{E}} \kappa_{\mathrm{E}}\left(P_{e k}(j), \underline{P}_{e}, \bar{P}_{e}, \Phi_{i, e k}(j)\right)
\end{aligned}
$$

where $\kappa_{\mathrm{L}}: \mathbb{R} \times \mathbb{R} \times \mathbb{R}_{+} \rightarrow \mathbb{R}$ and $\kappa_{\mathrm{E}}: \mathbb{R} \times \mathbb{R} \times \mathbb{R} \times$ $\mathbb{R}_{+} \rightarrow \mathbb{R} \cdot \hat{p}_{l k}$ is the predicted inelastic load at node $l$ and time $k$, and $\phi_{i, l k}(j)$ is the fractional contribution of elastic unit $i$ to this load at negotiation round $j$. $\Phi_{i, e k}(j)$ is the fractional contribution of unit $i$ to flow $P_{e k}(j)$. The forms chosen for the functions $\kappa_{\mathrm{L}}(\cdot, \cdot, \cdot)$ and $\kappa_{\mathrm{E}}(\cdot, \cdot, \cdot, \cdot)$ 
are discussed in the examples of section V. Convergence results are given in section IV-A.

6) Convergence check (price controller): The new price vector $\boldsymbol{\lambda}_{i}(j+1)$ is sent to each elastic participant; increment $j$ and return to step 2 unless the constraints (5b) and $(5 \mathrm{c})$ have been satisfied. Although the inequalities (5b) are satisfied in a finite number of steps, the power matches are reached asymptotically, so a tolerance $\varepsilon$ is chosen for the convergence check:

$$
\left|\frac{p_{l k}(j)}{\hat{p}_{l k}}-1\right| \leq \varepsilon, \quad l=1, \ldots, m_{\mathrm{i}}, \quad k=1, \ldots, T
$$

Qualitatively, the negotiation penalizes those at the wrong end of a congested line with lower prices, and generators receive lowered prices should there still be too much power scheduled to the loads. If the negotiation starts from relatively high initial prices, for example, more expensive generators will reduce their power output as the price is driven down, and the process converges to prices encouraging an efficient satisfaction of the loads. Through the scheduling action, storage units are able to perform arbitrage over price differentials as they emerge, thereby reducing price volatility.

While trying to maximize social welfare as defined in (5a), the scheme assumes no cooperation between producers or consumers, and only easily-verifiable information is required from them. We do, however, assume that participants can easily calculate their power schedule based on a set of prices over a discrete-time horizon, and that deviation from such an agreed schedule would be prevented by sufficient penalties. We also assume that price-inelastic power flows can be predicted perfectly over the horizon used.

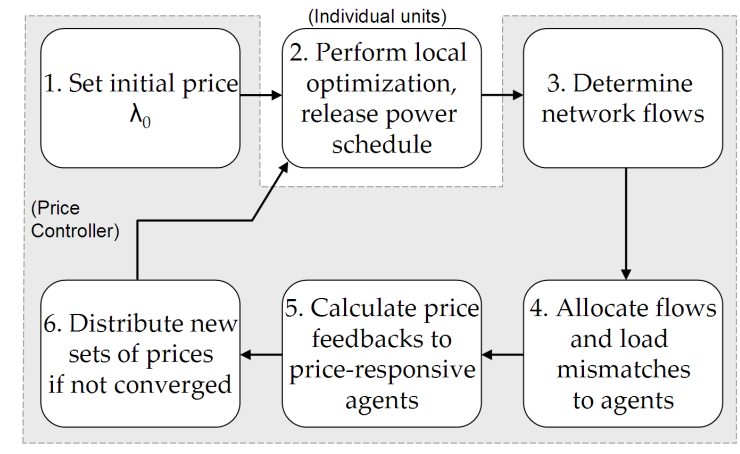

Fig. 2. Negotiation algorithm described in section III-A.

\section{B. Details of allocation algorithm}

There are several ways of assigning flows heuristically to units on a network, since in reality it is never possible to say whose generated power is being consumed elsewhere on a network, even though it is simple to determine the power flows in each line of the grid. $\mathrm{Ng}$ [15] and others have derived such algorithms, but these can lead to negative distribution factors, i.e. a generator can be given a negative responsibility for the flow in a given line. Practically it is more acceptable to use a method that gives only positive values, since in the price feedback step of equation (10) we want to punish constraint
TABLE I

ASSIGNMENT OF FLOWS AND LOAD SUPPLY TO GENERATORS FOR THE FLOWS SHOWN IN FIG. 3

\begin{tabular}{r|c|c|c|c} 
MW & Value & $G_{1}$ & $G_{2}$ & $G_{4}$ \\
\hline Flows: $1 \rightarrow 2$ & 517 & 517 & 0 & 0 \\
$1 \rightarrow 3$ & 483 & 483 & 0 & 0 \\
$3 \rightarrow 2$ & 183 & 60 & 0 & 123 \\
$4 \rightarrow 3$ & 1000 & 0 & 0 & 1000 \\
Loads: Node 2 & 1700 & 577 & 1000 & 123 \\
Node 3 & 1300 & 423 & 0 & 877
\end{tabular}

violation in an intuitive way. Therefore we choose a method due to Bialek [14].

Consider the network of Fig. 3, the shape of which is the same as used in [11]. For the line flow solution shown it is straightforward to issue the assignments shown in Table I. The algorithm is constructed from the assumption that power mixes proportionally between incoming and outgoing flows at each node. Now suppose that the flows are hypothetical and a lower demand is predicted at node 2 . This would require changes to generator outputs. From the entries in Table I we can compute $\phi_{1,2}=\frac{577}{1700}=0.34$, i.e. $G_{1}$ 's responsibility for mismatch at load 2, and issue an appropriate price control signal to $G_{1}$ using equation (10), and similarly calculate price signals for the other generators.

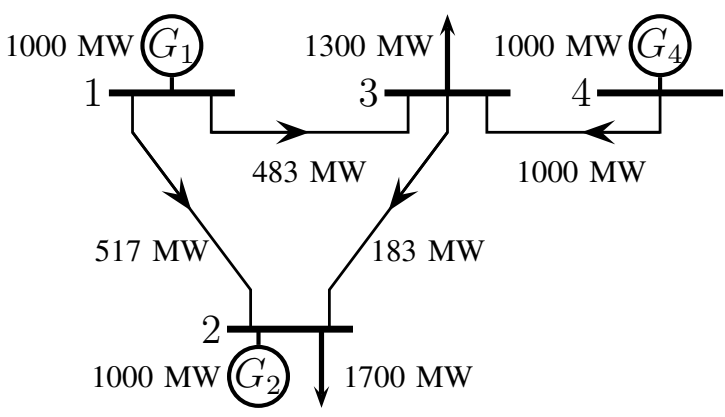

Fig. 3. Network flow state. Table I shows how these flows can be allocated to the generators on the network.

\section{CONVERGENCE AND OPTIMALITY CONDITIONS}

\section{A. Convergence}

The strategy in this approach to the Optimal Power Flow problem is that the problem of planning generators' schedules has been changed from a large-scale optimization into a problem of guaranteeing the convergence of a pricing algorithm. One aim is for such a price control scheme to allow action to be taken to force convergence such that a feasible solution always emerges from the procedure, in order to guarantee that operating constraints will be satisfied. As shown in section $\mathrm{V}-\mathrm{B}$, convergence has been demonstrated in complex cases with congested lines on a non-trivial network using such a controller.

For the uncongested case with each $\tilde{J}_{i}(\cdot)$ convex (i.e. $c_{i}>$ $\left.0, \forall i=1, \ldots, m_{\mathrm{e}}\right)$, and without storage units, convergence conditions for a negotiation with load imbalance feedback as in equation (14) were determined. Generation limits are assumed not to be hit. Although analysis of Bialek's algorithm 
is difficult and only just furthered in [16], if the blame coefficients $\phi_{i, l k}(j)$ are assumed equal, the following sufficient condition on the price feedback coefficient $K_{\mathrm{L}}$ for convergence to a power balance is reached:

$$
0<K_{\mathrm{L}}<2 m_{\mathrm{e}}\left[\sum_{i=1}^{m_{\mathrm{e}}} \frac{1}{c_{i}}\right]^{-1}
$$

In such a case, convergence to a power mismatch less than magnitude $\epsilon$ from a starting mismatch of magnitude $\epsilon_{0}$ is achieved, for $\beta:=\frac{K_{\mathrm{L}}}{m_{\mathrm{e}}} \sum_{i=1}^{m_{\mathrm{e}}} \frac{1}{c_{i}}$, in $N_{\text {conv }}$ steps, where

$$
N_{\text {conv }}=\left\lceil\frac{\ln \left(\epsilon / \epsilon_{0}\right)}{\ln |1-\beta|}\right\rceil \text {, }
$$

noting that both the numerator and denominator are negative. This means that for a given network population, convergence time has only a logarithmic dependence on the initial mismatch $\epsilon_{0}$. For succintness, proofs of these results are not given here, but are held along with further discussion at [17].

\section{B. Optimality}

In the example illustrated in section V-A, the nodes unaffected by congestion are shown to have the same price. When prices are equal across the network, convergence to a feasible solution satisfying $(5 \mathrm{c})$ implies optimality. This is because when the price is constrained to be equal amongst all loads, the assumptions of section IV-A imply that total power output during each time step is a strictly increasing linear function of price. Since there is only one load here, there is only one price that will satisfy this load. In other words, the feasible set in this optimization is a singleton if all generators themselves are acting optimally. In the more complex example of section V-B, however, no such optimality guarantee can be made at present.

It should be noted here, however, that continuous feasibility (in other words, reliability) of flows over a power network is more important than economic optimality, since network failure has extremely serious consequences. This is seen in present-day trading mechanisms, where the main priority is to establish a clearing price in the day-ahead market, and contingencies in real-time are regularly dealt with by telephone commands. In the current market even the objective function is a sum of private variables, so we cannot tell how optimal a dispatch has been reached, even before management costs and market power of the actors are considered.

Although convex cost functions have been considered in the example above, it is not required that all cost functions are convex for there to be a unique global optimal dispatch. However when non-convex cost functions are present there will be a duality gap for some optimal solutions. In other words, there will be no set of prices (dual variables) which will yield the global optimum. Indeed if enough of the units have 'bad' cost functions there may even be no set of prices that produces power flows satisfying the network constraints.

\section{EXAMPLE APPLICATIONS}

The negotiation procedure is shown in detail for a 4-bus network, so that the mechanics can be examined. Then success on a 39-bus network and storage effects are demonstrated.
TABLE II

TABLE OF NETWORK PARAMETERS FOR THE 4-BUS EXAMPLE

\begin{tabular}{c|c|c|c|c} 
Param. & Line 1-2 & Line 1-3 & Line 2-3 & Line 3-4 \\
\hline$\sigma_{i j}$ & $1 / 2$ & 1 & $1 / 3$ & $1 / 2$ \\
$\bar{P}_{e}$ & 1000 & 1000 & 1000 & 300 \\
$\underline{P}_{e}$ & 1000 & 1000 & 1000 & -300
\end{tabular}

TABLE III

TABLE OF GENERATOR PARAMETERS FOR THE 4-BUS EXAMPLE

\begin{tabular}{c|c|c|c|c} 
Param. & $G_{1}$ & $G_{2}$ & $G_{4}$ & Description \\
\hline$a_{i}$ & 0 & 0 & 0 & Fixed cost \\
$b_{i}$ & 2.5 & 2.0 & 1.7 & Linear cost \\
$c_{i}$ & 0.18 & 0.22 & 0.2 & Quadratic cost \\
$\bar{p}_{i}$ & 1000 & 1000 & 1000 & Upper power limit \\
$\underline{p}_{i}$ & 0 & 0 & 0 & Lower power limit \\
$\underline{\alpha}_{i}$ & 0.1 & 0.1 & 0.1 & Penalty for output changes \\
$p_{i}$ & 200 & 200 & 200 & Upper power change limit \\
$\underline{\Delta p}$ & -200 & -200 & -200 & Lower power change limit
\end{tabular}

\section{A. 4-bus network}

A simple network with four nodes is used to demonstrate the negotiation algorithm, as shown in Fig. 4. Three nodes support price-elastic generators, and the fourth node supports an inelastic load to be satisfied by the generators. Using the previously-defined notation, $m_{\mathrm{e}}=3, m_{\mathrm{i}}=1, m_{\mathrm{s}}=1$. A time step of $\tau=15$ minutes is used, with a time horizon of $T=24$ steps, i.e. 6 hours. This horizon is chosen on the grounds that in a practical implementation of such a power matching scheme, both demand and wind power outputs could be predicted effectively over the period [18].

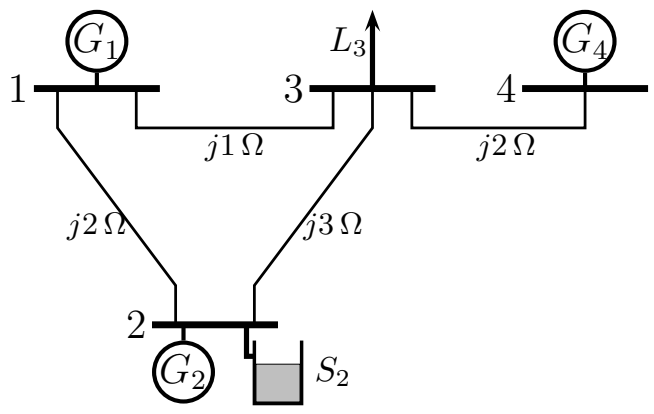

Fig. 4. 4-bus network topology with line impedances and unit placement.

Table II gives the parameters for the 4-bus network, and Table III gives the parameters for the price-elastic units. The storage unit at node 2 has a capacity of $100 \mathrm{MWh}$ and a maximum power magnitude of $200 \mathrm{MW}$, with midpoint deviation penalty $\gamma=0.12 € /(\mathrm{MWh})^{2}$.

For the price update rule in equation (10) we take:

$$
\kappa_{\mathrm{L}}\left(\hat{p}_{l k}, \tilde{p}_{l k}, \phi_{i, l k}\right)=K_{\mathrm{L}} \phi_{i, l k}\left(\hat{p}_{l k}-\tilde{p}_{l k}\right)
$$

and $\kappa_{\mathrm{E}}\left(P_{e k}, \underline{P}_{e}, \bar{P}_{e}, \Phi_{i, e k}\right)$

$$
=K_{\mathrm{E}} \Phi_{i, e k}\left(\max \left(\bar{P}_{e}-P_{e}, 0\right)-\max \left(P_{e}-\underline{P}_{e}, 0\right)\right)
$$

where $K_{\mathrm{L}}$ and $K_{\mathrm{E}}$ are positive scalars determining how heavily to act on load mismatches and line overflows respectively. The value of (15) is non-zero only when the line is congested in either direction. If the line is congested, unit $i$ will be penalised according to the proportion of the excess flow on that line for which it has been assigned responsibility. Here 
$K_{\mathrm{L}}=K_{\mathrm{E}}=0.075$ is used, consistent with the bounds given in section IV-A. Convergence to final prices at node 1 is shown over 15 steps in Fig. 6, and the final prices for all the priceelastic units are shown in Fig. 7. Fig. 8 shows how the load at node 3 is satisfied by the three generators plus storage unit; the storage unit is shown to take advantage of price differences across the horizon, buying at cheap times and selling at peak demand.

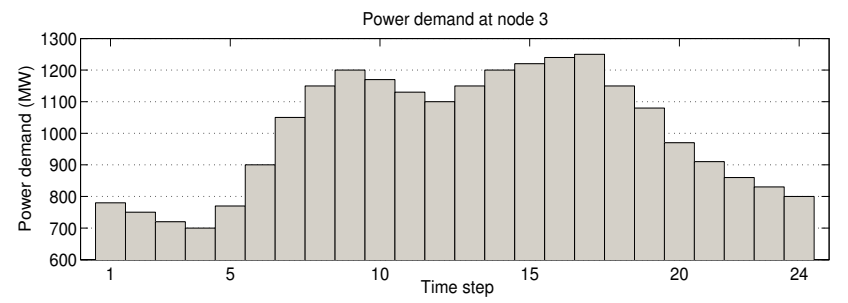

Fig. 5. 4-bus negotiation: Demand schedule for the load at node 3.

Price Schedule Evolution over Negotiation Process for Node 1

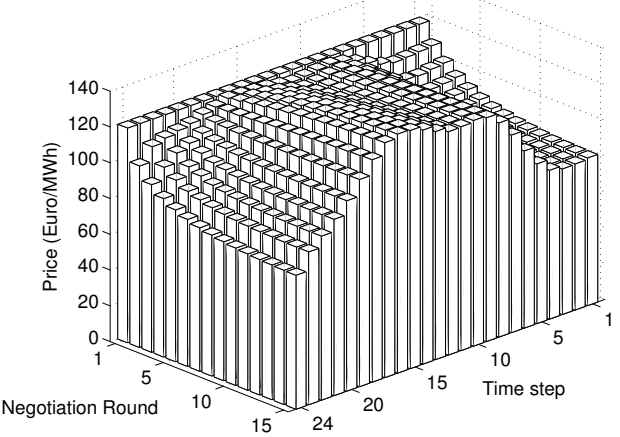

Fig. 6. 4-bus negotiation: Price schedule evolution over the negotiation procedure for node 1 .

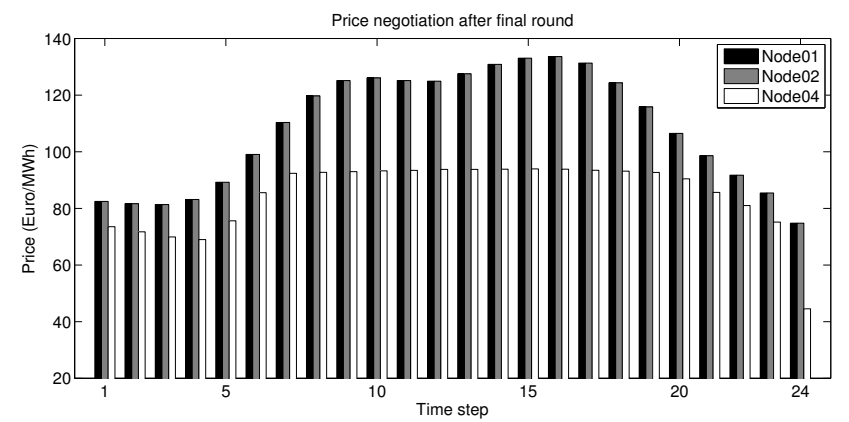

Fig. 7. 4-bus negotiation: Final prices for each price-elastic unit.

High prices coincide with times of high power demand, and the generator at node 4 receives a low price in comparison to othe generators, since the price controller gives a price disincentive that stops excess power overloading the line between nodes 3 and 4 . The bold line in Fig. 8 represents the gross power output of the generators and storage unit combined, and coincides as required with the demand profile of Fig. 5.

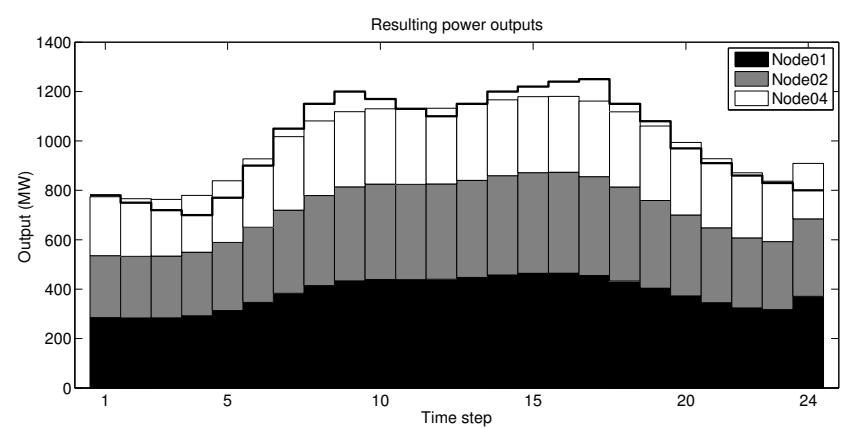

Fig. 8. 4-bus negotiation: Stacked power outputs showing how load at node 3 is satisfied. The bold line shows the storage flows relative to the sum of the generator outputs (the stacked bars).

\section{B. 39-bus network}

The negotiation procedure has also been employed on larger networks. As an example, the IEEE 39-bus network from [19] shown in Fig. 9 is used here. Price-elastic generators are placed at nodes 30-39, storage at nodes 31 and 38, and inelastic units at nineteen other nodes. For compactness, the unit and network parameters are not given here, but power constraints are set for each line at $450 \mathrm{MW}$. The negotiation gives a flow satisfying each of the loads' schedules to within $2 \%$ within 50 iterations. Fig. 10 shows the final price schedule for the nodes, and Fig. 11 shows the breakdown of the total power provision between the price-sensitive units. Fig. 10 shows that nodes 33 and 34 experience particularly low prices since any power not drawn by the load at node 20 is transported entirely by the single cosntrained line 16-19.

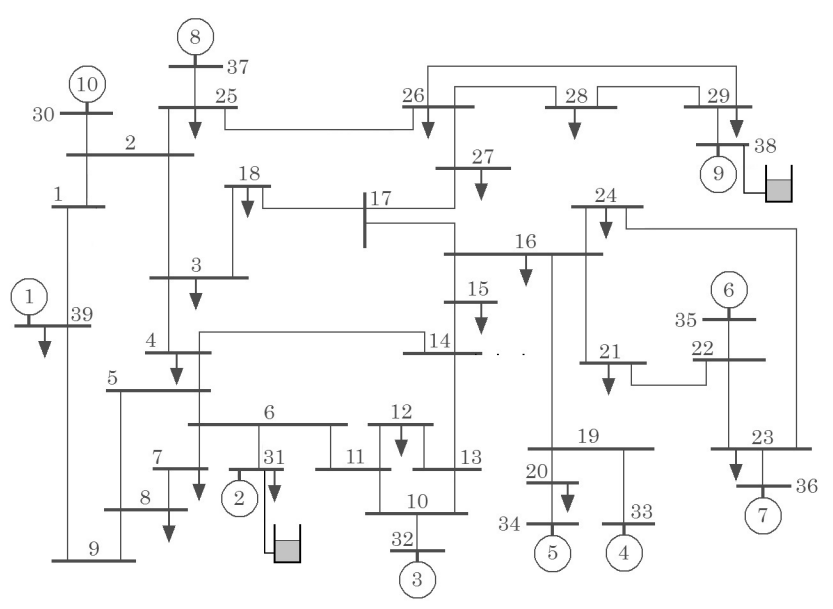

Fig. 9. 39-bus negotiation: Network topology showing populated nodes.

\section{Effect of storage}

The same 39-bus scenario was used to test the effect of storage. Table IV shows how use of storage affects total generation costs and nodal prices. The storage is split equally between nodes 31 and 38. Although total generation costs and average prices did not change significantly, price volatility (measured by the standard deviation of the nodal price from its average over the time horizon) is significantly decreased by the introduction of storage. 


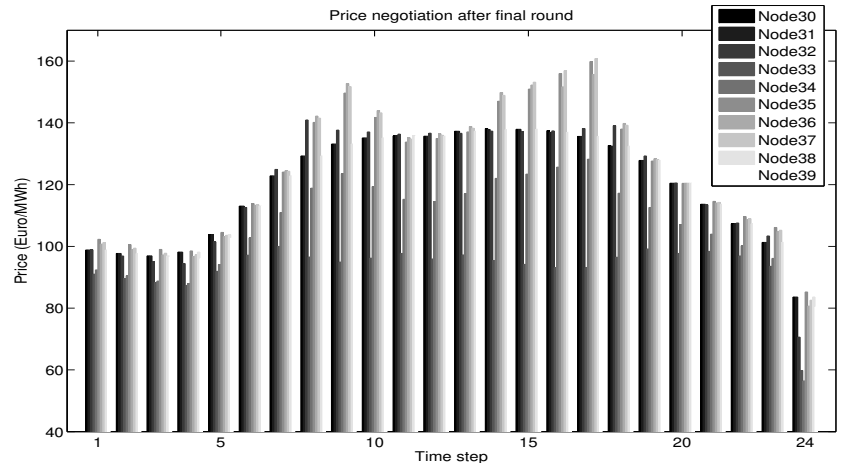

Fig. 10. 39-bus negotiation: Final price schedule.

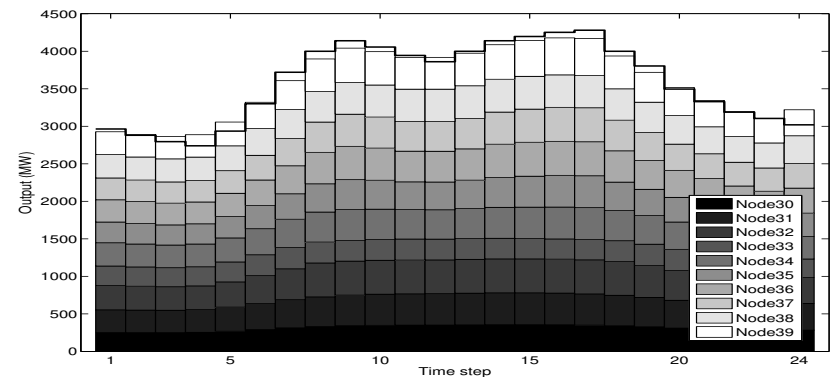

Fig. 11. 39-bus negotiation: Gross power breakdown. The bold line shows the storage flows relative to the sum of the generator outputs (the stacked bars).

TABLE IV

EFFECT OF STORAGE ON DISPATCH COST AND PRICE VOLATILITY

\begin{tabular}{c|c|c|c}
$\begin{array}{c}\text { Total storage } \\
(\mathrm{MWh})\end{array}$ & $\begin{array}{c}\text { Dispatch cost } \\
(\mathrm{M} €)\end{array}$ & $\begin{array}{c}\text { Average price, } \\
\text { node 38 }(€ / \mathrm{MWh})\end{array}$ & $\begin{array}{c}\text { Price s.d., node } \\
38(€ / \mathrm{MWh})\end{array}$ \\
\hline 0 & 3.76 & 118.25 & 20.87 \\
200 & 3.73 & 119.45 & 13.47 \\
400 & 3.71 & 119.34 & 12.00
\end{tabular}

\section{IMPLEMENTATION REQUIREMENTS}

The ICT requirements of the control scheme are straightforward to obtain and reasonable. Part of the control scheme is inherently distributed over the participants. The centralized price controller only needs to receive power profiles from each unit at every negotiation iteration $j$. Profiles can be issued from 'smart' aggregators of domestic devices present at a node, equivalent to a price-elastic unit. From this information, the controller computes the new nodal price profiles for all nodes and transmits them back. The only information the participants need is the vector of length $T$ nodal prices over the negotiation horizon and some means of predicting their power needs. The storage agents moreover need a measurement of their level of storage. Each elastic or storage agent needs to solve a problem of dimension $T$ at each negotiation round, which means the longest allowed computation period is the real price update sampling period divided by the maximum number of rounds. This means the number of iterations for acceptable convergence need to be bounded. The flow equations can be solved by the central price controller or by microcontrollers at each node, as described in Appendix A. The price controller needs to assign responsibilities to nodes and compute the control law (10) for constraint violations.

\section{CONCLUSIONS}

A negotiation-based predictive pricing algorithm has been employed to produce feasible power flows under selfish operation of network agents. All price sensitive network agents participate in the negotiation process in order to reduce power peaks and price volatility. Price insensitive network agents are modeled as predictable load profiles, such that price sensitive network agents can optimize their production/consumption. Simulation results show that load matching and line constraints are satisfied for large networks populated by elastic, inelastic and storage components. Analytical convergence results are given for the algorithm under a strong set of assumptions.

As well as continued analysis of convergence and optimality properties, several extensions to the scheme are planned. Of particular interest are reserve margins, which can be priced in the same framework by bringing the reserves into the local constraints. Constraint (2b) would be changed and the reserve price would appear in the local cost function. Each participant then trades off reserve and real power offered. Reserves needed are set by the grid operator, e.g. for $N-1$ reliability. Since at present reserves are set conservatively and in far advance to manage a wide range of contingencies [2], significant savings could be made by using up-to-date state information to set reserve levels more intelligently on a receding horizon basis.

\section{REFERENCES}

[1] William R. Hughes and Andrew Parece. The economics of price spikes in deregulated power markets. The Electricity Journal, 15(6):31-44, July 2002

[2] P.P.J. van den Bosch, A. Jokic, J. Frunt, W.L. Kling, F. Nobel, P. Boonekamp, W. de Boer, and R.M. Hermans. Incentives-based ancillary services for power system integrity. In Energy Market, 2009. EEM 2009. 6th International Conference on the European, pages 1-7, 2009.

[3] Hadi Saadat. Power System Analysis. McGraw-Hill Education (India) Pvt Ltd, 2002.

[4] Arthur R. Bergen and Vijay Vittal. Power Systems Analysis. Pearson, 2006.

[5] F.C. Schweppe, R.D. Tabors, J.L. Kirtley, H.R. Outhred, F.H. Pickel, and A.J. Cox. Homeostatic utility control. Power Apparatus and Systems, IEEE Transactions on, PAS-99(3):1151-1163, 1980.

[6] H. Glavitsch and F. Alvarado. Management of multiple congested conditions in unbundled operation of a power system. In Power Industry Computer Applications., 1997. 20th International Conference on, pages 374-380, 1997.

[7] Fernando L. Alvarado. Is system control entirely by price feasible? In Hawaii International Conference on System Sciences, volume 2, page 53b, Los Alamitos, CA, USA, 2003. IEEE Computer Society.

[8] J. C. Jacobo and D. De Roure. A decentralised DC optimal power flow model. In Electric Utility Deregulation and Restructuring and Power Technologies, 2008. DRPT 2008. Third International Conference on, page $484490,2008$.

[9] A.J. Conejo and J.A. Aguado. Multi-area coordinated decentralized DC optimal power flow. Power Systems, IEEE Transactions on, 13(4):12721278, 1998.

[10] S.J. Wang, S.M. Shahidehpour, D.S. Kirschen, S. Mokhtari, and G.D. Irisarri. Short-term generation scheduling with transmission and environmental constraints using an augmented lagrangian relaxation. Power Systems, IEEE Transactions on, 10(3):1294-1301, 1995.

[11] A. Jokic, M. Lazar, and P. P. J. van den Bosch. Real-time control of power systems using nodal prices. International Journal of Electrical Power and Energy Systems, 31(9):522530, 2009.

[12] Andre Jokic. Price-Based Optimal Control of Electrical Power Systems. $\mathrm{PhD}$ thesis, TU Eindhoven, 2007.

[13] R.D. Christie, B.F. Wollenberg, and I. Wangensteen. Transmission management in the deregulated environment. Proceedings of the IEEE, 88(2):170-195, 2000. 
[14] J Bialek. Tracing the flow of electricity. IEE Proc.-Gener. Transm. Distrib., 143(4):313-320, 1996.

[15] W. Y. Ng. Generalized generation distribution factors for power system security evaluations. IEEE Transactions on Power Apparatus and Systems, page 10011005, 1981.

[16] C. Achayuthakan, C.J. Dent, J.W. Bialek, and W. Ongsakul. Electricity tracing in systems with and without circulating flows: Physical insights and mathematical proofs. Power Systems, IEEE Transactions on, 25(2):1078-1087, 2010.

[17] Joseph Warrington. Proof of convergence conditions for paper Predictive power dispatch through negotiated locational pricing http://tinyurl.com/34yrs5z (Alternatively by contact with the author), 2010.

[18] Gregor Giebel, Paul Sorensen, and Hannele Holttinen. Forecast error of aggregated wind power. Technical report, TradeWind, 2007.

[19] M.A. Pai. Energy Function Analysis for Power System Stability. Springer, 1 edition, 1989.

\section{APPENDIX}

\section{Distributed determination of power flow}

The solution of network flows can be framed as the optimization problem (16), under the assumptions of section II-A:

$$
\min _{\delta} J=\sum_{i=1}^{n} J_{i}=\sum_{i=1}^{n}\left[G_{i}-L_{i}+V^{2} \sum_{j \in \mathcal{N}_{i}} \sigma_{i j}\left(\delta_{j}-\delta_{i}\right)\right]^{2}
$$

where $\delta=\left[\delta_{1} \ldots \delta_{n}\right]^{\mathrm{T}}$ is the vector of $n$ nodal phase angles, $G_{i}$ and $L_{i}$ are the generation (power injection) and load (power extraction) at node $i$, and $\mathcal{N}_{i}$ is the set of all nodes directly connected to node $i . \sigma_{i j}$ is the susceptance for lines connecting node $j \in \mathcal{N}_{i}$ to node $i$, with $j \notin \mathcal{N}_{i} \Leftrightarrow \sigma_{i j}=0$. Clearly when the objective function $J=0$ the power balance at each node is satisfied and we have a solution to the flows. The algorithm used is the following:

1) For each node $i$, if $J_{i}<\epsilon^{2}$, set a convergence flag for node $i$. Unset if node $i$ no longer satisfies this.

2) Set the phase angle $\delta_{i}$ such that power balance is satisfied for that node. This is given by:

$$
\begin{aligned}
0 & =\frac{G_{i}-L_{i}}{V^{2}}+\sum_{j \in \mathcal{N}_{i}} \sigma_{i j}\left(\delta_{j}-\delta_{i}\right) \\
\Rightarrow \delta_{i} & =\left[\sum_{j \in \mathcal{N}_{i}} \sigma_{i j}\right]^{-1}\left(\frac{G_{i}-L_{i}}{V^{2}}+\sum_{j \in \mathcal{N}_{i}} \sigma_{i j} \delta_{j}\right)
\end{aligned}
$$

Note that applying rule (17) only requires infomation on the local phase angles $\left\{\delta_{j} \mid j \in \mathcal{N}_{i}\right\}$, and that the reciprocal of the sum can always be taken since every node $i$ has at least one neighbour.

Repeat steps 1 and 2 sequentially for each node.

3) If all nodes' flags are set, the algorithm has converged to within a total imbalance of magnitude less than $n \epsilon$; terminate the procedure. Otherwise return to step 1.

Convergence is reliable in practice, but an analysis is outside this paper's scope. If no comprehensive model of the network even exists, as with the European UCTE, then a decentralized solution based on local microcontrollers has advantages, particularly if the initial guess, taken from the previous state, is already near to the correct solution. A solution for the nonlinear equations, i.e. avoiding the approximation $\sin \left(\delta_{j}-\delta_{i}\right) \approx\left(\delta_{j}-\delta_{i}\right)$, is also possible in the same way, except that (17) is replaced by a term involving phasor addition of the sinusoids at adjacent nodes.

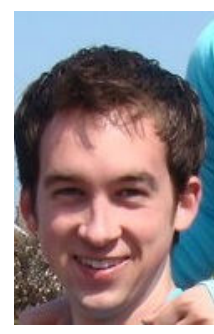

Joseph Warrington (M'10) received the B.A. and M.Eng. degrees from Cambridge University, England in 2008. His masters thesis was on active control of the earth's climate. In the summer of 2007 he studied agent-based collision avoidance algorithms in the Control and Dynamical Systems laboratory at the California Institute of Technology. From 2008-9 he worked on wind turbine control for Wind Technologies Ltd., in Cambridge. He is now a Ph.D. student in the Automatic Control Laboratory at ETH Zurich, where his interests include control on electricity networks and distributed optimization.

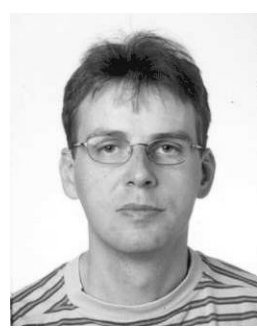

Sébastien Mariéthoz (M'05) received the Ph.D. degree in electrical engineering for his work on asymmetrical multilevel converters from EPFL, Lausanne, Switzerland in 2005. He was research fellow with the PEMC group, University of Nottingham, UK, focusing on the design and control of new matrix converter topologies. He joined the Automatic Control Laboratory, ETH Zürich in 2006, where he is currently senior researcher. His current research interests include optimal design and control of power electronic systems, model predictive control and

power systems control.

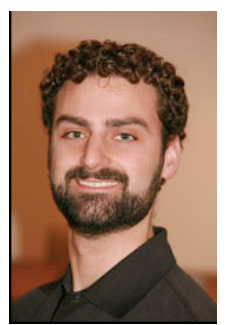

Colin N. Jones received his bachleur and master of applied science at the University of British Columbia in Canada in 2001 before obtaining his $\mathrm{PhD}$ from the University of Cambridge in 2005. Colin has been a post-doctoral researcher in the Automatic Control Laboratory at the ETH in Zurich since 2006 and a senior researcher from 2008. He founded the company Apex Optimization focusing on scheduling and optimization of human resources in 2007. His current research interests are in the areas of highspeed predictive control, computational geometry, energy management and optimal scheduling.

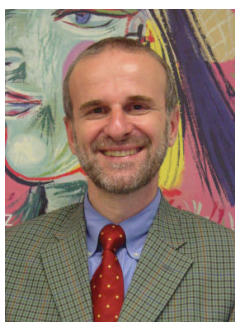

Manfred Morari (F'05) was appointed head of the Automatic Control Laboratory at ETH Zurich in 1994. Before that he was the McCollum-Corcoran Professor of Chemical Engineering and Executive Officer for Control and Dynamical Systems at the California Institute of Technology. He obtained the diploma from ETH Zurich and the Ph.D. from the University of Minnesota, both in chemical engineering. His interests are in hybrid systems and the control of biomedical systems.

In recognition of his research contributions, he received numerous awards, among them the Donald P. Eckman Award and the John R. Ragazzini Award of the Automatic Control Council, the Allan P. Colburn Award and the Professional Progress Award of the AIChE, the Curtis W. McGraw Research Award of the ASEE, Doctor Honoris Causa from BabesBolyai University, Fellow of IEEE and IFAC, the IEEE Control Systems Field Award, and was elected to the National Academy of Engineering (U.S.).

Manfred Morari has held appointments with Exxon and ICI plc and serves on the technical advisory boards of several major corporations. 